IPM/P-2001/002

\title{
T-duality and Noncommutative DBI Action
}

\author{
Davoud Kamani \\ Institute for Studies in Theoretical Physics and Mathematics (IPM) \\ P.O.Box: 19395-5531, Tehran, Iran \\ e-mail:kamani@theory.ipm.ac.ir
}

\begin{abstract}
In this article we study the noncommutative description of the DBI Lagrangian and its T-dual counterpart. We restrict the freedoms of the noncommutativity parameters of these Lagrangians. Therefore the noncommutativity parameter, the effective metric, the effective coupling constant of the string and the extra modulus $\tilde{\Phi}$ of the effective T-dual theory, can be expressed in terms of the closed string variables $g, B, g_{s}$ and the noncommutativity parameter of the effective theory of open string.
\end{abstract}




\section{Introduction}

Over the past years there have been much activities exploring the relation between string theory and noncommutative geometry [1, 2, 3, 4. There have been attempts to explain noncommutativity on D-brane worldvolume through the study of open strings in the presence of background fields [3, 4. For slowly varying fields, the effective Lagrangian of the open string theory is the Dirac-Born-Infeld (DBI) Lagrangian [5]. The equivalence of noncommutative and ordinary DBI theory was proven [1]. There are some general descriptions for the open string effective action such that noncommutativity parameter is arbitrary. In other words, a general DBI theory has been proposed to be described by a noncommutative action including an extra modulus $\Phi$ [1]. The modules and their T-dualities have been considered [6, 7], and the relation to the DBI Lagrangian has been explored [7]. Other aspects of the noncommutative DBI theory have been discussed in Ref. [8].

We observe that the open string metric and the noncommutativity parameter appear as the background fields of the T-dual theory of string theory. Also the background fields of string theory appear as the effective metric and the noncommutativity parameter of the T-dual theory of the effective theory.

We concentrate on the freedoms of the noncommutative DBI action and its T-dual counterpart. The freedoms of the noncommutativity parameters will be restricted. In this case the noncommutativity parameter, the effective metric, the effective coupling constant of the

string and the modulus $\tilde{\Phi}$ of the effective T-dual theory are expressed in terms of the closed string variables $g, B, g_{s}$ and the noncommutativity parameter of the effective theory of open string.

This paper is organized as follows. In section 2, we give a brief review of the T-duality of the string action with the background fields $g$ and $B$. In section 3, the noncommutative descriptions of the open string effective action and its T-dual theory will be discussed. In section 4, relations between the effective variables of the noncommutative DBI Lagrangian and the variables of its T-dual counterpart will be discussed.

\section{String action and its T-duality}

A fundamental bosonic string ending on a $\mathrm{D}_{p}$-brane with the background fields $g$ and $B$ has the action [9]

$$
S=\frac{1}{4 \pi \alpha^{\prime}} \int_{\Sigma} d^{2} \sigma\left(g_{i j} \partial_{a} X^{i} \partial^{a} X^{j}-2 \pi i \alpha^{\prime} \epsilon^{a b} B_{i j} \partial_{a} X^{i} \partial_{b} X^{j}\right)
$$


where $\Sigma$ is the string worldsheet. Just like Ref. [1], we assume that the target space and the string worldsheet are Euclidean, and $B_{i j}$ is non-zero only for $i, j=1, \ldots, r$ and $g_{i j}$ vanishes for $i=1, \ldots, r, j \neq 1, \ldots, r$, where $r \leq p+1$. Further more assume $g_{i j}$ and $B_{i j}$ to be constant background fields.

Now consider the T-duality of the theory. In the case of toroidal compactification, when $d$-spatial coordinates are compactified on the torus $T^{d}$, the T-duality group is $O(d, d ; \mathbf{Z})[10]$. Assume that the $\mathrm{D}_{p}$-brane is wrapped on the torus $T^{p}$. A particular element of $O(p, p ; \mathbf{Z})$ T-duality group is

$$
T=\left(\begin{array}{cc}
\mathbf{0} & \mathbf{1}_{p \times p} \\
\mathbf{1}_{p \times p} & \mathbf{0}
\end{array}\right) .
$$

Under the action of this element of the T-duality group, we have the following transformation for the background fields $g$ and $B$ [11

$$
\left(g+2 \pi \alpha^{\prime} B\right) \rightarrow\left(\tilde{g}+2 \pi \alpha^{\prime} \tilde{B}\right)=\left(g+2 \pi \alpha^{\prime} B\right)^{-1} .
$$

In other words, T-duality transformations of $g$ and $B$ are

$$
\begin{aligned}
& \tilde{g}=\left(g+2 \pi \alpha^{\prime} B\right)^{-1} g\left(g-2 \pi \alpha^{\prime} B\right)^{-1}, \\
& \tilde{B}=-\left(g+2 \pi \alpha^{\prime} B\right)^{-1} B\left(g-2 \pi \alpha^{\prime} B\right)^{-1},
\end{aligned}
$$

where $\tilde{g}$ is symmetric and $\tilde{B}$ is antisymmetric. These are background fields of the T-dual theory. Also the string coupling $g_{s}$ transforms as

$$
\tilde{g}_{s}=\frac{g_{s}}{\sqrt{\operatorname{det}\left(g+2 \pi \alpha^{\prime} B\right)}} .
$$

The action of the dual theory can be written as the following

$$
\tilde{S}=\frac{1}{4 \pi \alpha^{\prime}} \int d^{2} \sigma\left(\tilde{g}_{i j} \partial_{a} \tilde{X}^{i} \partial^{a} \tilde{X}^{j}-2 \pi i \alpha^{\prime} \epsilon^{a b} \tilde{B}_{i j} \partial_{a} \tilde{X}^{i} \partial_{b} \tilde{X}^{j}\right)
$$

The equation of motion and the boundary conditions resulted from this action imply that $\left\{\tilde{X}^{i}\right\}$ are coordinates on a noncommutative space.

\section{Noncommutativity of the effective theories}

Now let us discuss the noncommutative description of the DBI Lagrangian and the T-duality of it. The noncommutative description can be expressed in terms of noncommutative gauge field and the open string variables $G_{(0)}, \theta_{0}$ and $G_{s}^{(0)}$ [1]

$$
\widehat{\mathcal{L}}_{(0)}=\frac{1}{(2 \pi)^{p}\left(\alpha^{\prime}\right)^{\frac{p+1}{2}} G_{s}^{(0)}} \sqrt{\operatorname{det}\left(G_{(0)}+2 \pi \alpha^{\prime} \widehat{F}\right)},
$$


where the open string variables are

$$
\begin{aligned}
& G_{(0)}^{i j}=\left(\left(g+2 \pi \alpha^{\prime} B\right)^{-1} g\left(g-2 \pi \alpha^{\prime} B\right)^{-1}\right)^{i j}, \\
& G_{(0) i j}=\left(\left(g-2 \pi \alpha^{\prime} B\right) g^{-1}\left(g+2 \pi \alpha^{\prime} B\right)\right)_{i j}, \\
& \theta_{0}^{i j}=-\left(2 \pi \alpha^{\prime}\right)^{2}\left(\left(g+2 \pi \alpha^{\prime} B\right)^{-1} B\left(g-2 \pi \alpha^{\prime} B\right)^{-1}\right)^{i j}, \\
& G_{s}^{(0)}=g_{s}\left(\frac{\operatorname{det} G_{(0)}}{\operatorname{det}\left(g+2 \pi \alpha^{\prime} B\right)}\right)^{\frac{1}{2}} .
\end{aligned}
$$

These equations and the equations (4) and (5) give the following results

$$
\begin{aligned}
& G_{(0)}=\tilde{g}, \\
& \theta_{0}=\left(2 \pi \alpha^{\prime}\right)^{2} \tilde{B},
\end{aligned}
$$

i.e. the open string metric and the noncommutativity parameter appear as the background fields of the T-dual theory of string theory.

The T-dual description of the Lagrangian (8) can be obtained from (8) by the parameters $\tilde{G}_{s}^{(0)}, \tilde{G}_{(0)}$ and $\tilde{\theta}_{0}$. These parameters obey the equations $(9)$ which the parameters $g_{s}, g$ and $B$ should be changed by the parameters $\tilde{g}_{s}, \tilde{g}$ and $\tilde{B}$ respectively. Therefore similar to the relations (10), we obtain

$$
\begin{aligned}
& \tilde{G}_{(0)}=g, \\
& \tilde{\theta}_{0}=\left(2 \pi \alpha^{\prime}\right)^{2} B,
\end{aligned}
$$

that is the background fields of the string theory appear as the effective metric and the noncommutativity parameter of the effective theory of the T-dual theory.

There is a two-form $\Phi$ such that for the effective theory more general description with an arbitrary parameter $\theta$ is possible [1]. In this case the effective Lagrangian is

$$
\widehat{\mathcal{L}}=\frac{1}{(2 \pi)^{p}\left(\alpha^{\prime}\right)^{\frac{p+1}{2}} G_{s}} \sqrt{\operatorname{det}\left(G+2 \pi \alpha^{\prime}(\Phi+\widehat{F})\right)},
$$

where the variables $G, \Phi$ and $G_{s}$ depend on $g, B$ and $\theta$,

$$
\begin{gathered}
\frac{1}{G+2 \pi \alpha^{\prime} \Phi}=-\frac{\theta}{2 \pi \alpha^{\prime}}+\frac{1}{g+2 \pi \alpha^{\prime} B} \\
G_{s}=g_{s}\left(\frac{\operatorname{det}\left(G+2 \pi \alpha^{\prime} \Phi\right)}{\operatorname{det}\left(g+2 \pi \alpha^{\prime} B\right)}\right)^{\frac{1}{2}} .
\end{gathered}
$$


Similarly there is such freedom for the effective Lagrangian of the dual theory.

Since the noncommutativity parameter of the T-dual theory of (12) i.e. $\tilde{\theta}$ is independent of the variables of the original theory, i.e. $g, B$ and $g_{s}$, and also it is independent of the parameter $\theta$, the T-dual theory of (12) partially depends on the original theory and its effective theory, but not completely. We shall introduce an appropriate and consistent relation such that the partially dependence changes to the completely dependence. That is, $\tilde{\theta}$ will be expressed in terms of $g, B$ and $\theta$.

\section{Relations between the effective variables}

In the effective action and in the T-dual theory of it, there are two arbitrary noncommutativity parameters $\theta$ and $\tilde{\theta}$. These parameters appear in the variables $G, \Phi, \tilde{G}$ and $\tilde{\Phi}$ and also in the effective string couplings $G_{s}$ and $\tilde{G}_{s}$. We can express one of them in terms of the other. For this, we introduce the following relation

$$
\tilde{G}+2 \pi \alpha^{\prime} \tilde{\Phi}=\left(g+2 \pi \alpha^{\prime} B\right)^{-1}\left(G-2 \pi \alpha^{\prime} \Phi\right)\left(g-2 \pi \alpha^{\prime} B\right)^{-1} .
$$

From the equation (13) and its T-dual analogue and the equation (15) we obtain

$$
\tilde{\theta}=-\left(g-2 \pi \alpha^{\prime} B\right) \theta\left(g+2 \pi \alpha^{\prime} B\right),
$$

which implies, if the effective theory is noncommutative (ordinary) the effective theory of the T-dual theory also is noncommutative (ordinary) and vice-versa. Equation (15) has interesting properties that we present some of them.

As expected, equation (15) also holds under the following exchanges

$$
\begin{aligned}
G & \leftrightarrow \tilde{G}, \\
\Phi & \leftrightarrow \tilde{\Phi}, \\
g & \rightarrow \tilde{g}, \\
B & \rightarrow \tilde{B} .
\end{aligned}
$$

To see this property, perform the transposed of both sides of the equation (15) and then use the equation (3).

Consider the commutative case i.e. $\theta=0$. Equation (16) also gives $\tilde{\theta}=0$. Therefore there are $G=g, \Phi=B, \tilde{G}=\tilde{g}$ and $\tilde{\Phi}=\tilde{B}$. According to these, the equation (15) reduces to the duality equation (3), which is an expected result. 
The following values for the variables $\theta, G$ and $\Phi$ (for the maximal rank $r=p+1$ )

$$
\begin{aligned}
& \theta=B^{-1}, \\
& G=-\left(2 \pi \alpha^{\prime}\right)^{2} B g^{-1} B, \\
& \Phi=-B,
\end{aligned}
$$

satisfy the equation (13) [1]. Also consider the dual variables with the forms

$$
\begin{aligned}
& \tilde{\theta}=\tilde{B}^{-1}=-\left(g-2 \pi \alpha^{\prime} B\right) B^{-1}\left(g+2 \pi \alpha^{\prime} B\right), \\
& \tilde{G}=-\left(2 \pi \alpha^{\prime}\right)^{2} \tilde{B} \tilde{g}^{-1} \tilde{B}=-\left(2 \pi \alpha^{\prime}\right)^{2}\left(g+2 \pi \alpha^{\prime} B\right)^{-1} B g^{-1} B\left(g-2 \pi \alpha^{\prime} B\right)^{-1}, \\
& \tilde{\Phi}=-\tilde{B}=\left(g+2 \pi \alpha^{\prime} B\right)^{-1} B\left(g-2 \pi \alpha^{\prime} B\right)^{-1},
\end{aligned}
$$

where $\tilde{g}$ and $\tilde{B}$ in terms of $g$ and $B$ are used. These parameters satisfy the T-dual analogue of the equation (13). Therefore for the solutions (18) and (19), the equation (15) reduces to an identity.

According to the equation (15), we can express the dual variables $\tilde{G}$, $\tilde{\Phi}$ and $\tilde{G}_{s}$ in terms of the parameters $g, B, \theta$ and $g_{s}$. Using the fact that for a matrix $M$ there is the identity $\operatorname{det} M=\operatorname{det} M^{T}$, we obtain

$$
\tilde{G}_{s}=\frac{\tilde{g}_{s}}{g_{s}} G_{s}=\frac{G_{s}}{\sqrt{\operatorname{det}\left(g+2 \pi \alpha^{\prime} B\right)}},
$$

which implies that the quantity $\frac{G_{s}}{g_{s}}$ is T-duality invariant. In other words $\frac{g_{Y M}}{\sqrt{g_{s}}}$ is invariant, i.e.

$$
\tilde{g}_{Y M}=g_{Y M} \sqrt{\frac{\tilde{g}_{s}}{g_{s}}}=g_{Y M}\left[\operatorname{det}\left(g+2 \pi \alpha^{\prime} B\right)\right]^{-\frac{1}{4}} .
$$

The dual variables $\tilde{G}$ and $\tilde{\Phi}$ are symmetric and antisymmetric parts of the right hand side of the equation (15), i.e.

$$
\begin{aligned}
& \tilde{G}=\left(g+2 \pi \alpha^{\prime} B\right)^{-1} G\left(g-2 \pi \alpha^{\prime} B\right)^{-1}, \\
& \tilde{\Phi}=-\left(g+2 \pi \alpha^{\prime} B\right)^{-1} \Phi\left(g-2 \pi \alpha^{\prime} B\right)^{-1},
\end{aligned}
$$

where $G$ and $\Phi$ are given by the equation (13). The equations (22) and (23) for $\theta=0$, reduce to the results $\tilde{G}=\tilde{g}$ and $\tilde{\Phi}=\tilde{B}$. They also respect the exchanges (17), and for the solutions (18) and (19) change to identities.

Consider the interesting special case $\theta=\theta_{0}$. From the equation (16) we obtain

$$
\tilde{\theta}=\tilde{\theta}_{0}=\left(2 \pi \alpha^{\prime}\right)^{2} B
$$


Therefore the antisymmetric tensors $\Phi$ and $\tilde{\Phi}$ vanish which means, if the freedom of the parameter $\theta$ removes, the freedom in the T-dual theory also removes. For this special case the effective metric $\tilde{G}$ and the effective coupling constant $\tilde{G}_{s}$ reduce to

$$
\begin{aligned}
& \tilde{G}=\tilde{G}_{(0)}, \\
& \tilde{G}_{s}=\tilde{G}_{s}^{(0)}=\frac{g_{s}}{\sqrt{\operatorname{det} g}} .
\end{aligned}
$$

Note that according to the equations (20) and (22) the quantity $\frac{\sqrt{\operatorname{det} G}}{G_{s}^{2}}$ is T-duality invariant.

\section{Conclusion}

We saw that when the extra modulus $\Phi$ is zero (i.e. $\theta=\theta_{0}$ ), the background fields of string theory (T-dual of string theory), are the effective metric and the noncommutativity parameter of the effective T-dual theory (the effective theory of string theory).

We introduced a consistent relation between the open string variables $(G, \Phi)$ and the dual variables $(\tilde{G}, \tilde{\Phi})$. Therefore the noncommutativity parameters are expressed in terms of each other. In this case if the effective theory is noncommutative (ordinary), the effective T-dual theory also is noncommutative (ordinary) and vice-versa. The ratio of the effective open string coupling to the open string coupling is a T-duality invariant quantity. In the effective theory of the T-dual theory the noncommutativity parameter, the effective metric, the effective coupling of string and the antisymmetric tensor $\tilde{\Phi}$ are expressed in terms of the closed string variables $g, B, g_{s}$ and the noncommutativity parameter $\theta$.

\section{Acknowledgement}

The author would like to thank H. Arfaei for useful discussion.

\section{References}

[1] N. Seiberg and E. Witten, JHEP 9909(1999)032, hep-th/9908142.

[2] A. Connes, M.R. Douglas and A. Schwarz, JHEP 9802(1998)003, hep-th/9711162; M.R. Douglas and C. Hull, JHEP 9802(1998)008, hep-th/9711165; F. Ardalan, H. Arfaei and M.M. Sheikh-Jabbari, JHEP9902(1999)016, hep-th/9810072; A. Fayyazuddin and M. Zabzine, Phys. Rev. D62(2000)046004, hep-th/9911018; P.M. Ho and Y.T. Yeh, Phys. Rev. Lett. 85(2000)5523, hep-th/0005159; Y.E. Cheung and M. Krog, 
Nucl. Phys. B528(1998)185, hep-th/9803031; D. Bigatti and L. Susskind, Phys. Rev. D62(2000)066004, hep-th/9908056; A. Schwarz, Nucl. Phys. B534(1998)720, hepth/9805034.

[3] C.S. Chu and P.M. Ho, Nucl. Phys. B550(1999)151, hep-th/9812219; Nucl. Phys. B568(2000)447, hep-th/9906192.

[4] V. Schomerus, JHEP 9906(1999)030, hep-th/9903205; F. Ardalan, H. Arfaei and M.M. Sheikh-Jabbari, Nucl. Phys. B576(2000)578, hep-th/9906161; N. Ishibashi, "A Relation between Commutative and Noncommutative Descriptions of Dbranes", hep-th/9909176.

[5] E.S. Fradkin and A.A. Tseytlin, Phys. Lett. B163(1985)123; C.G. Callan, C. Lovelace, C.R. Nappi and S.A. Yost, Nucl. Phys. B288(1987)525-550; A. Abouelsaood, C.G. Callan, C.R. Nappi and S.A. Yost, Nucl. Phys. B280(1987)599.

[6] B. Morariu and B. Zumino, "Super Yang - Mills on the Noncommutative Torus", hep-th/9807198; D. Brace and B. Morariu, JHEP 9902(1999)004, hep-th/9810185; D. Brace, B. Morariu and B. Zumino, Nucl. Phys. B545(1999)192, hep-th/9810099; Nucl. Phys. B549(1999)181, hep-th/9811213.

[7] C. Hofman and E. Verlinde, JHEP 9812(1998)010, hep-th/9810116; Nucl. Phys. B547(1999)157, hep-th/9810219; B. Pioline and A. Schwarz, JHEP 9908(1999)021, hep-th/9908019.

[8] R. Garousi, Nucl. Phys. B579(2000)209, hep-th/9909214; L. Cornalba, JHEP 0009(2000)017, hep-th/9912293; T. Lee, Phys. Lett. B478(2000)313, hep-th/9912038; J. Pawelczyk, JHEP 0008(2000)006, hep-th/0003057; S. Ryang, "Open String and Morita Equivalence of the Dirac-Born-Infeld Action with Modulus $\Phi "$, hep-th/0003204.

[9] J. Dai, R.G. Leigh and J. Polchinski, Mod. Phys. Lett. A4(1989)2073; R. Leigh, Mod. Phys. Lett. A4(1989)2767.

[10] K.S. Narain, H. Sarmadi and E. Witten, Nucl. Phys. B279(1987)369.

[11] A. Giveon, M. Porrati and E. Robinovici, Phys. Rep. C244(1994)77-202, hepth/9401139; R.G. Leigh, Mod. Phys. Lett.A4 28(1989)2767. 